\title{
Heterogeneity of DNA distribution in diploid cells: a new predicitive discriminant factor for solid tumour behaviour
}

\author{
Jacques Assailly ${ }^{\mathrm{a}, *}$, Arnaud Desgrippes ${ }^{\mathrm{b}}$, \\ Brigitte Loridon-Rosa ${ }^{\mathrm{c}}$, Dominique Piron ${ }^{\mathrm{c}}$, \\ Roger Dachez ${ }^{\mathrm{d}}$ and Daniel Beurton ${ }^{\mathrm{b}}$ \\ a A.I.P.C. Laboratoire d'Analyse d'Images en \\ Pathologie Cellulaire, Centre HAYEM, Hôpital Saint \\ Louis, 1 ave C. Vellefaux, 75475 Paris Cedex 10, \\ France \\ b Service d'Urologie, Hôpital Ambroise PARE, \\ 92100 Boulogne, France \\ ${ }^{\mathrm{c}}$ Centre de Pathologie, 19 rue de Passy, 75016 Paris, \\ France \\ d Institut A. Fournier, 24 Bd Saint Jacques, \\ 75014 Paris, France
}

Received 6 July 1998

Revised 28 January 1999

Accepted 27 March 1999

Spatial nuclear DNA heterogeneity distribution of Feulgenstained DNA diploid cells was studied by image cytometry in voided urine of 119 patients without bladder tumour ( $n=$ $20)$ and with initial $(n=23)$ or previous $(n=76)$ diagnosed bladder tumour. For each patient, repetitive DNA measurements were performed during 1-4 years of follow up. Only cells of diploid DNA histograms and diploid subpopulations of aneuploid DNA histograms were used for analysis. DNA heterogeneity distribution of these diploid cells was quantified by statistical parameters of each nuclear optical density distribution. Discriminant analysis was performed on three groups of DNA histograms. Group A $(n=44)$ : aneuploid DNA histograms of patients with bladder tumour. Group D ( $n=55$ ): 38 diploid DNA histograms of the 20 patients without bladder tumour (subgroup D1) and 17 diploid DNA histograms of patients with a non-recurrent bladder tumour (subgroup D2). Group R $(n=27)$ : diploid DNA histograms of patients with bladder tumour recurrence. No statistically significant discriminant function was found to separate D1 and D2. However, the first canonical discriminant function C1 differentiated diploid cells of diploid DNA histograms (group D and group R) from diploid cell subpopulations of aneuploid DNA histograms (group A). Mean C1 values were
1.06, 0.84 and -1.45 for groups R, D and A, respectively. The second canonical discriminant function $\mathrm{C} 2$ differentiated diploid DNA histograms of patients with bladder tumour recurrence (group R) from diploid DNA histograms of patients without bladder tumour or without bladder tumour recurrence (group D). Mean C2 values were 1.78 and -0.76 for groups R and D, respectively. In $95 \%$ confidence limit, the rate of rediscrimination using the two first canonical discriminant functions $\mathrm{C} 1$ and $\mathrm{C} 2$ were $86.4,74.5$ and $74.1 \%$ for groups A, D and R, respectively. Percent of "grouped" cases correctly classified was $78.6 \%$. Thus spatial DNA heterogeneity distribution of diploid cells seems to quantitate probable genetic instability as a function of clinical evolution such as tumour recurrence, and suggests the possible presence of aneuploid stemlines in a heterogeneous tumour, even if a diploid DNA histogram is observed in a single sample. From standardized $\mathrm{C} 1$ and $\mathrm{C} 2$ canonical discriminant function coefficients, a DNA heterogeneity index $(2 \mathrm{c}-\mathrm{HI})$ is proposed to characterize diploid cells providing a descriptive and predictive discriminant factor for solid tumour behaviour.

Keywords: DNA content, ploidy, solid tumours, image cytometry, heterogeneity, bladder cancer

\section{Introduction}

For several years, measurements of DNA content or ploidy status (i.e., identifying diploid vs. aneuploid tumours) have been shown to be relevant for diagnosis, prognosis, and treatment of patients with solid tumours. Usually, aneuploidy has been found to correlate with increasing tumour grade and stage and with a worse prognosis as well as with recurrence than survival $[1,2,6,15]$.

By definition, diploid tumours have a "normal" amount of DNA while aneuploid tumours have an

\footnotetext{
*Corresponding author: A.I.P.C. Laboratoire d'Analyse d'Images en Pathologie Cellulaire, Centre HAYEM, Hôpital Saint Louis, 1 ave C. Vellefaux, 75475 Paris cedex 10, France. Tel.: + 330153722181 ; Fax: + 33015372185 .
} 
"abnormal" amount of DNA. In fact, when referring to a diploid tumour, quantitative DNA analysis (flow and image cytometry) measures gross differences in DNA content and does not detect chromosomal abnormalities such as translocations or deletion. The clinical behaviour of malignant cells seems to be strongly correlated to their ploidy status [11]. However, DNA diploid content is an unclear indication of premalignant change or malignancy potential, and, moreover, it has been observed that ploidy status can change during tumour evolution and treatment. Although diploid tumours with apparently normal DNA content have a better prognosis than DNA aneuploid tumours, recurrences were observed in many diploid solid tumours, particularly in more than half of recurrent bladder cancers [5]. In cases of prior tumour diagnosis, and especially in detection of premalignant or borderline lesions, diploid DNA content is not very useful either in routine clinical practice or in terms of individual prognosis and survival for each patient during follow up of their tumour.

Finally, intratumoural heterogeneity in DNA distribution patterns and genetic evolution, which have recently been described in some solid tumours [9,12-14, 16], suggests the possible presence of aneuploid stemlines in heterogenous tumours, even if a diploid DNA histogram is observed in a single sample of the tumour.

The purpose of this study was to evaluate spatial nuclear DNA heterogeneity distributions in nuclei of DNA diploid cells, as a means of identifying a reliable individual malignant precursor index, when genetic instability induces relatively small DNA alterations in relation to total DNA content, at the current level of quantitative DNA content detection by image analysis. We have shown that spatial DNA heterogeneity distribution parameters of diploid cells could identify diploid stemlines from diploid cells forming part of aneuploid stemlines and discriminate between different clinical states, such as, for example, patients with diploid bladder tumour recurrence.

\section{Materials and methods}

\subsection{Patient characteristics}

Between 1994 and 1997, 442 DNA-ploidy measurements on voided urine cells were performed on 119 patients followed up in the Urology Department of Ambroise PARE hospital. 23 patients had an initial diagnosed bladder tumour, 76 had a previous diagnosed bladder tumour, and 20 had no bladder tumour. For each urine DNA measurement, clinical status, conventional cytology, fibroscopy and histology were registered. During this period, one to 6 DNA-ploidy measurements were obtained for each patient at each fibroscopy or scheduled clinical controls.

Several groups of DNA content measurements were selected according to their DNA-ploidy status and well known clinical status. Group A $(n=44)$ consisted of aneuploid DNA histograms of patients with bladder tumour. Group D $(n=55)$ included 38 diploid DNA histograms of the 20 patients without bladder tumour (subgroup D1) and 17 diploid DNA histograms of patients with a non-recurrent bladder tumour (subgroup D2). Diploid DNA histograms of patients with a non-recurrent bladder tumour were selected as the second diploid histogram of three consecutive diploid DNA measurements with normal endoscopic control at a three month time interval. Group $\mathrm{R}(n=27)$ consisted of diploid DNA histograms of patients with bladder tumour recurrence. To test the classification obtained by discriminant analysis, an independent sample $\mathrm{A}^{\prime}(n=29), \mathrm{D}^{\prime}(n=36), \mathrm{R}^{\prime}(n=18)$ of DNA histogram groups A, D, R, respectively, was selected from urine sample of patients analysed at the Pathology Center during 1997 and 1998.

\subsection{Preparation of samples for image cytometry}

Following recommendations described in the ESACP report on standardization of DNA image cytometry [3], cellular DNA content measurements were determined by image analysis using a SAMBA 2005 cytometer (TITN-ALCATEL) and routinely processed Feulgen stained cytocentrifuged prepaparations of urine sediments. Rat liver hepatocytes were used as external standard. The coefficient of variation of the reference cell population never exceeded $6 \%$.

\subsection{DNA measurements and statistical analysis}

From the Feulgen slide, the image of each nucleus was digitized by a video camera in a series of units called pixels. To each pixel is assigned an amount of light converted in optical density (OD). Correlated by the Beer Lambert Law, the integrated optical density (IOD) of the $N$ nuclei pixels gives the DNA amount of the cell. For each nucleus, surface (SURF), Integrated Optical Density (IOD), Mean Optical Density (MOD), and statistical parameters of the OD distribution (Standard Error (ODSE), Skewness (ODSKE), 


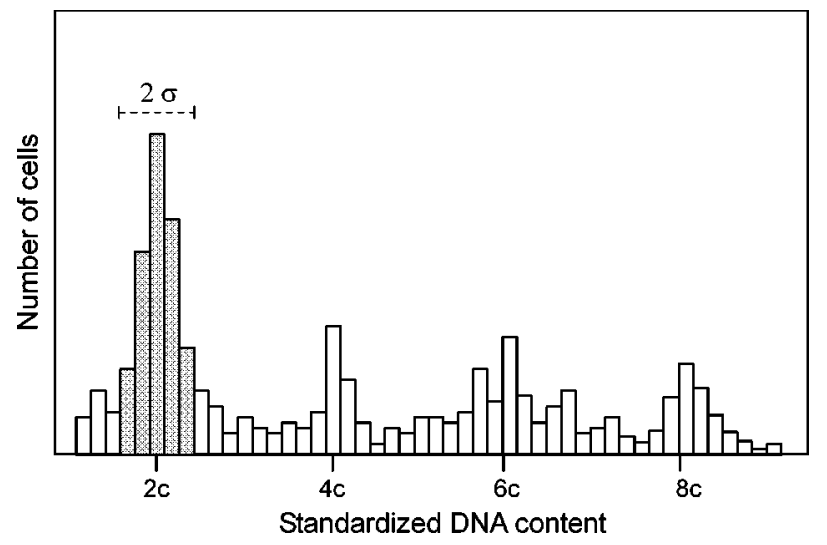

Fig. 1. Selection of diploid DNA cells $2 \mathrm{c}$ stemline reference of rat hepatocytes.

Table 1

Statistical parameters used to quantitate DNA heterogeneity of diploid cells

\begin{tabular}{lccccc}
\hline & VAR $^{a}$ & SE $^{a}$ & CV $^{a}$ & SKE $^{a}$ & KU $^{a}$ \\
\hline${ }^{*}$ SURF & var_surf & se_surf & cv_surf & ske_surf & kur_surf \\
${ }^{*}$ IOD & var_iod & se_iod & cv_iod & ske_iod & kur_iod \\
${ }^{*}$ MOD & var_mod & se_mod & cv_mod & ske_mod & kur_mod \\
${ }^{*}$ ODSE & var_odse & se_odse & cv_odse & ske_odse & kur_odse \\
${ }^{*}$ ODSKE & var_odske & se_odske & cv_odske & ske_odske & kur_odske \\
${ }^{*}$ ODKUR & var_odkur & se_odkur & cv_odkur & ske_odkur & kur_odkur \\
\hline${ }^{*}$ Parameters obtained from optical density for each nucleus of diploid cells. \\
${ }^{a}$ Statistical parameters relate to selected diploid cell populations of each patient. \\
\multicolumn{7}{l}{ Heavy type parameters: variables selected for canonical discriminant analysis. }
\end{tabular}

Kurtosis (ODKUR)) were calculated. The stochiometric relation between staining intensity and DNA content (IOD) was used to generate DNA histograms and select diploid cell subpopulations.

Diploid cell populations of each diploid DNA histogram and diploid subpopulations of aneuploid DNA histograms were selected in a $2 \sigma$ range calculated from the $2 \mathrm{c}$ stemline reference of rat hepatocytes (Fig. 1). This selection was performed to avoid possible interactions induced by heterogeneity of nuclear DNA content in a subpopulation of DNA diploid cells.

Spatial DNA heterogeneity distribution parameters were obtained for each selected diploid cell population by calculating the variance $\sigma^{2}$ (VAR), standard error (SE), coefficient of variation (CV), skewness (SKE) and kurtosis (KUR) of SURF, IOD, MOD, ODSE, ODSKE, ODKUR (Table 1). A minimum threshold of thirty selected diploid cells on each DNA histogram was used. Mean values of SURF, IOD, MOD, ODSE, ODSKE, ODKUR, were not used in canonical discriminant analyses to take into account only DNA heterogenity distribution parameters. Statistical analyses were first performed on these parameters with canonical discriminant analysis tools of SPSS.

\section{Results}

Canonical discriminant analysis on DNA heterogeneity distribution parameters was first performed on the two diploid DNA histogram subgroups D1 (patients without bladder tumour) and D2 (patients without bladder recurrence) of group D. No statistically significant discriminant function was found to separate D1 and D2.

Successive canonical discriminant analyses were at once performed on selected diploid cells of 126 DNA histograms of the three groups A, D (D1 + D2), and $\mathrm{R}$, to eliminate DNA heterogeneity distribution parameters (variables) which have non significant statistical contributions for discrimination. A 0.30 absolute correlation threshold between each variable and any discriminant function was used. Twelve of the 36 vari- 


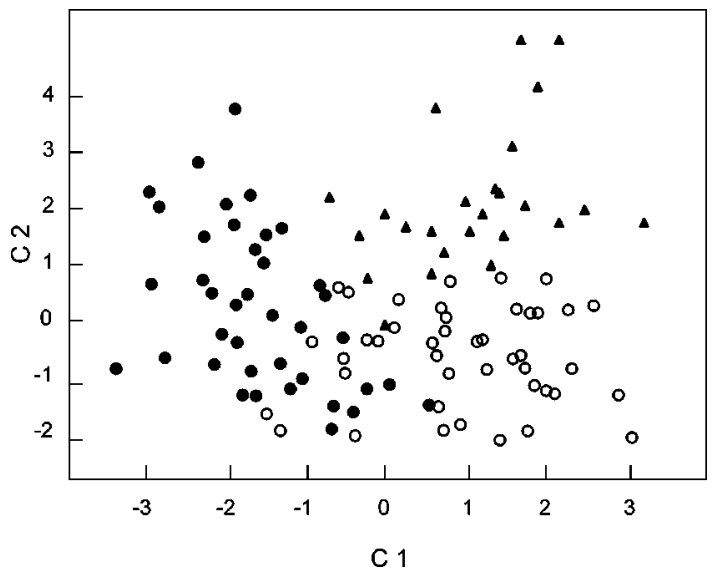

Fig. 2. Scattergram of 126 cases obtained by canonical discriminant analysis on DNA heterogeneity distribution parameters of diploid cells. C1 and C2, first and second canonical functions respectively, are clustered in three separate territories corresponding to groups D (o): diploid DNA histograms $(n=55)$ of patients without bladder tumour or without bladder tumour recurrence, $\mathrm{A}(\bullet)$ : aneuploid DNA histograms of patients with bladder tumour $(n=44)$ and $\mathrm{R}(\mathbf{\Delta})$ : diploid DNA histograms of patients with bladder tumour recurrence.

ables were thus selected (Table 1) in the following results.

Figure 2 is a scattergram of 126 cases obtained by canonical discriminant analysis, where $\mathrm{C} 1$ and $\mathrm{C} 2$ are the first and the second canonical functions, respectively. The cases are apparently clustered in three separate territories. The canonical discriminant function C1 differentiated diploid cells from diploid DNA histograms (groups D and R) from diploid cell subpopulations of aneuploid DNA histograms (group A). Mean C1 values were $1.06,0.84$ and -1.45 for groups $R, D$ and $\mathrm{A}$, respectively.

The canonical discriminant function $\mathrm{C} 2$ was also able to separate diploid DNA histograms of patients with bladder tumour recurrence (group R) from diploid DNA histograms of patients without bladder tumour or without tumour recurrence (group D). Mean C2 values were 1.78 and -0.76 for groups $R$ and $D$, respectively.

Separation of the three groups A, D, R by both $\mathrm{C} 1$ and $\mathrm{C} 2$ axis, was not ambiguous, although it had slightly overlapping nearness borderlines. In $95 \%$ confidence limit, the mean percentage of "grouped" cases correctly classified was $78.6 \%$ (Table $2 \mathrm{a}$ ). When $\mathrm{A}^{\prime}, \mathrm{D}^{\prime}$ and $\mathrm{R}^{\prime}$ independent groups were reclassified with standardized canonical discriminant function coefficients, the percentage of "grouped" cases correctly classified was $76.6 \%$ (Table 2b).

Among the 12 of 36 variables (DNA heterogeneity distribution parameters) used for discrimination of $\mathrm{D}$,
Table 2

a. Classification results of groups D, A and R

\begin{tabular}{|c|c|c|c|c|}
\hline \multirow[b]{2}{*}{ Group } & \multirow{2}{*}{$\begin{array}{l}\text { No. of } \\
\text { cases }\end{array}$} & \multicolumn{3}{|c|}{ Predicted group membership } \\
\hline & & $\mathrm{D}(\%)$ & $\mathrm{A}(\%)$ & $\mathrm{R}(\%)$ \\
\hline $\mathrm{D}$ & 55 & $41(74.5)$ & $11(20.0)$ & $3(5.5)$ \\
\hline A & 44 & $6(13.6)$ & $38(86.4)$ & $0(0)$ \\
\hline $\mathrm{R}$ & 27 & $3(11.1)$ & $3(14.8)$ & $20(74.1)$ \\
\hline \multicolumn{5}{|c|}{ b. Reclassification results of independent groups $\mathrm{D}^{\prime}, \mathrm{A}^{\prime}$ and $\mathrm{R}$} \\
\hline \multirow[b]{2}{*}{ Group } & \multirow{2}{*}{$\begin{array}{c}\text { No. of } \\
\text { cases }\end{array}$} & \multicolumn{3}{|c|}{ Predicted group membership } \\
\hline & & $\mathrm{D}(\%)$ & $\mathrm{A}(\%)$ & $\mathrm{R}(\%)$ \\
\hline $\mathrm{D}^{\prime}$ & 36 & $27(75.0)$ & $4(11.1)$ & $5(13.8)$ \\
\hline $\mathrm{A}^{\prime}$ & 29 & $3(10.3)$ & $24(82.8)$ & $2(6.9)$ \\
\hline $\mathrm{R}^{\prime}$ & 18 & $2(11.1)$ & $3(16.6)$ & $13(72.2)$ \\
\hline
\end{tabular}

$\mathrm{R}$ and $\mathrm{A}$ groups, the four with the largest statistical contributions were cv_iod, cv_surf, ske_iod, kur_iod for C1 and kur_surf, ske_surf, se_odse, cv_mod for $\mathrm{C} 2$.

DNA heterogeneity distribution parameters seem to allow characterization and quantitation of abnormal instability of diploid cells both for tumour identification and for differentiating two clinical conditions such as tumour recurrence. From these results, standardized $\mathrm{C} 1$ and $\mathrm{C} 2$ canonical discriminant function coefficients can be used as co-ordinates of a DNA heterogeneity index (2c-HI) for diploid cell differentiation in the different canonical discriminant analysis territories. In this study, we have observed that in $27 \%$ of cases, only DNA aneuploid histograms in urine sediments predict bladder tumour recurrence, althought conventional cytology remains normal [4]. When, during follow-up, 2c-HI DNA heterogeneity index was used to characterize the different registered diploid DNA histograms obtained from patients with bladder tumour, the percentage of predicted recurrence increased from 27 to $52 \%$.

\section{Discussion}

Numerous studies correlating abnormalities of cellular DNA content with clinical behaviour have been reported [5-15]. Aneuploidy is a well recognised feature of human solid tumours, but its clinical significance has been hampered by the poor evolution of some DNA diploid malignant tumours [8]. Cancer progression is associated with increasing chromosomal anomalies that can be grossly assessed by measuring tumour cell DNA content. It has been shown 
that, when minor genomic or chromosomal abnormalities are not taken into account by DNA content measurements, chromatin fine structure could differentiate DNA diploid carcinomas [10]. The present study was designed to validate, on a long follow-up of bladder cancer patients, the heterogeneity of diploid cell DNA distribution as a predictive discriminant factor for solid tumours.

The 2c-HI DNA heterogeneity index of diploid cells, obtained from canonical discriminant analysis of parameters describing variance within the DNA nuclear distributions of each cell, has been shown to allow quantitation of probable genetic instability of these diploid cells. The results reported in the present study on diploid cells are in agreement with those obtained on chromatin texture analysis $[7,10]$. Stability of DNA heterogeneity distribution parameters calculated from $2 \sigma$ range diploid cells, can be evaluated on the 38 repetitive diploid histograms obtained from 20 patients without bladder tumour measurements during followup. Whatever inherent differences in measurement's conditions (staining, standardization, different analyser), these 38 diploid histograms are well grouped in diploid group D. This suggests that, through detection of modifications of diploid DNA heterogeneity distribution parameters (i.e., predicted group membership) in different repetitive DNA histograms registered over a period of time, progression or regression of bladder tumour, could be significantly predicted.

For each clinical application, both for tumour detection and for prognosis (progression or evaluation of recurrence risk), a discriminant analysis on retrospective data pools of DNA ploidy measurements could be performed to determine thresholds of 2c-HD index co-ordinates ( $\mathrm{C} 1$ and $\mathrm{C} 2$ functions) according to the required discrimination. Each patient with a series of diploid DNA histograms could be followed up by monitoing multiple 2c-HD index values to obtain individual predictive factor.

Thus, if a $2 \mathrm{c}-\mathrm{HI}$ index of a diploid histogram is in the $\mathrm{C} 1, \mathrm{C} 2$ co-ordinate range of $2 \mathrm{c}-\mathrm{HI}$ index of diploid subpopulations of aneuploid DNA histograms, or of diploid histograms corresponding to tumour recurrence, this may suggest possible future clinical evolution. From several apparently identical diploid DNA histograms, results on bladder tumours have pointed out the ability of heterogeneity of DNA distribution to discriminate different tumour behaviours.

This method could be applied to detection of precancerous lesions in which slight alteration of DNA content occurs without modification of the total amount of
DNA. During repeated DNA examinations performed over time on each with bladder tumour patient, we observed different DNA ploidy status. Aneuploidy or diploidy status of a tumour can change during clinical evolution and treatment. It is not, as frequently reported in the past, an immutable specific characteristic. In our study of bladder tumours followed up during 1-4 years, $49 \%$ of patients had changed their DNAploidy status; $46 \%$ had changed from aneuploidy to diploidy, $23 \%$ from diploidy to aneuploidy and 30\% had alternated three times [4]. These variations in DNA ploidy status are certainly induced either by intratumoural heterogeneity or by reversible DNA repair or DNA impairing, which slightly affects DNA content of some diploid cells, without possible detection of signifiant DNA content variation by image analysis.

In the case of numerous heterogeneous tumours, knowledge of DNA ploidy status on biopsy or single histological section cannot ensure the absence of aneuploid cell populations in another part of the tumour. Multiple-site sampling is very important for determination of DNA ploidy patterns, because some tumours having heterogeneous DNA content can be erroneously diagnosed as diploid when only a single sample is examined. In a preliminary study, we also observed diploid and aneuploid DNA histograms in a few series of sequential tumour sections in breast cancer. Indices of 2c-HD obtained on diploid imprints, were well correlated with the presence of aneuploid DNA histograms on more distant sections.

In conclusion, it can be expected that DNA heterogeneity analysis of diploid cell populations, will renew the interest of DNA ploidy in solid tumours by obtaining both a non-binary diploid-aneuploid result and an individual predictive discriminant factor for tumour patients.

\section{References}

[1] J. Assailly, J.D. Monet, Y. Moustafa and G. Brugal, Contribution of quantitative cytology and ploidy in the diagnostic and the survey of bladder tumour. Study of 52 cases by image analyser Samba, J. Urol. 95(1) (1989), 3-9.

[2] J. Assailly, A. Viellefond, G. Benoit, D. Schoevaert and E. Martin, Evaluation of DNA image cytometry on 84 histologically investigated bladder tumours, Urol. Res. 20(6) (1992), 457458.

[3] A. Böcking, F. Giroud and A. Reith, Consensus report of ESACP task force on standardization of DNA image cytometry, Anal. Cell. Pathol. 8 (1995), 67-74.

[4] A. Desgrippes, Intérêt de la plö̈die dans les tumeurs superficielles de la vessie, Thèse de Médecine, Université Paris 7, 1997. 
[5] Y. Fradet, Markers of prognosis in superficial bladder cancer, Seminars in Urology 10(1) (1992), 28-38.

[6] M.L. Friedlander, D.W. Hedley and I.W. Taylor, Clinical and biological significance of aneuploidy in human tumours, J. Clin. Pathol. 37 (1984), 961-974.

[7] G. Haroske, K. Friedrich, F. Theissig, W. Meyer and K.D. Kunze, Heterogeneity of the chromatin fine structure in DNAdiploid breast cancer cells, Anal. Cell. Pathol. 8 (1995), 213 226.

[8] C.J. Herman, Cytometric DNA analysis in the management of cancer, Cancer 69 (1992), 1553-1556.

[9] G. Ljungberg, C. Mehle, R. Stenling and G. Roos, Heterogeneity in renal cell carcinoma and its impact on prognosis - a flow cytometric study, Br. J. Cancer 74 (1996), 123-127.

[10] F. Liautaud Roger, J.R. Teyssier, D. Ferre, J. Dufer and P. Coninx, Can chromatin texture predict structural kayotipic changes in diploid cells from thyroid cold nodules, Anal. Cell. Pathol. 4(6) (1992), 421-428.
[11] D.E. Merkel and W.L. McGuire, Ploidy, proliferative activity and prognosis. DNA flow cytometry of solid tumours, Cancer 65 (1990), 1194-1205.

[12] H. Nenning, J. Raßler and D.H. Minh, Heterogeneity of DNA distribution pattern in renal tumours, Anal. Cell. Pathol. 14(1) (1997), 9-17.

[13] M.N. Raber, B. Barlogie and J. Latreille, Ploidy, proliferative activity and estrogen receptor content in human breast, Cytometry 3 (1982), 36-41.

[14] S.E. Shackney and T.V. Shankey, Common patterns of genetic evolution in human solid tumours, Cytometry 29(1) (1997), 127.

[15] D. Seckinger, E. Sugarbaker and O. Frankfurt, DNA content in human cancer, Arch. Pathol. Lab. Med. 113 (1989), 612-626.

[16] R.P. Wersto, R. Liblit, D. Deitch and L.G. Koss, Variability in DNA measurements in multiple tumour samples of human colonic carcinoma, Cancer 67 (1991), 106-115. 


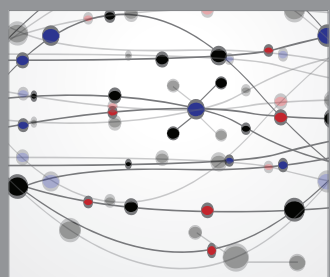

The Scientific World Journal
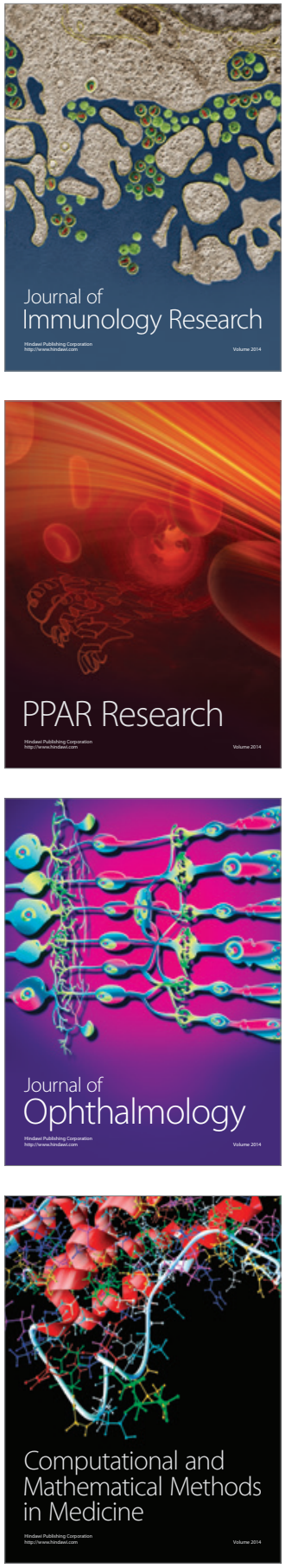

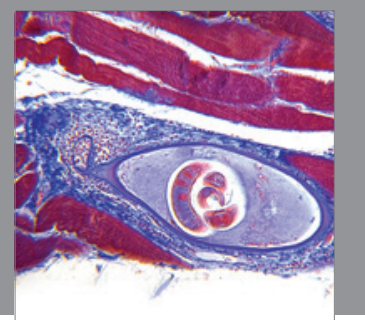

Gastroenterology

Research and Practice
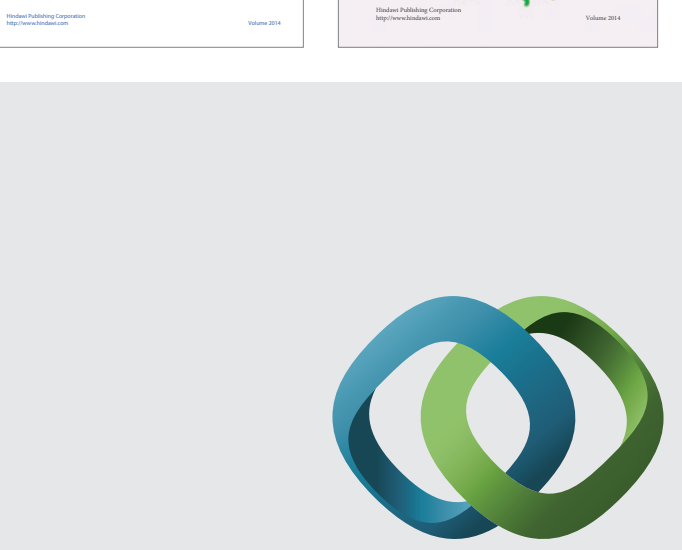

\section{Hindawi}

Submit your manuscripts at

http://www.hindawi.com
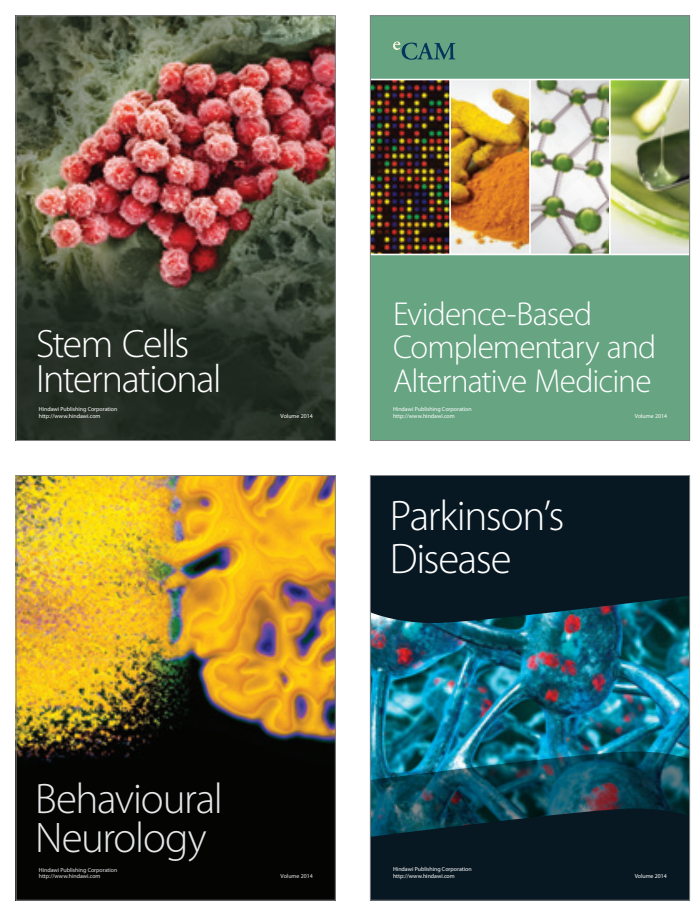

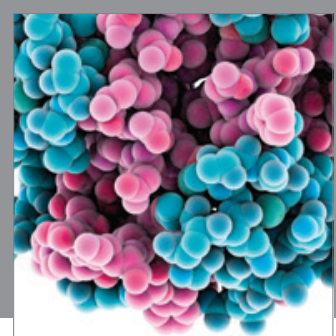

Journal of
Diabetes Research

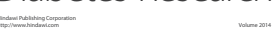

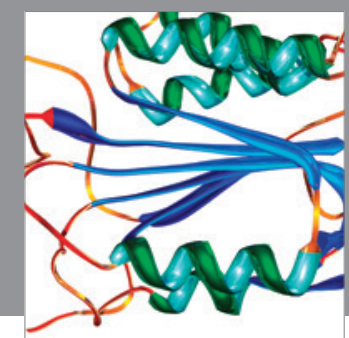

Disease Markers
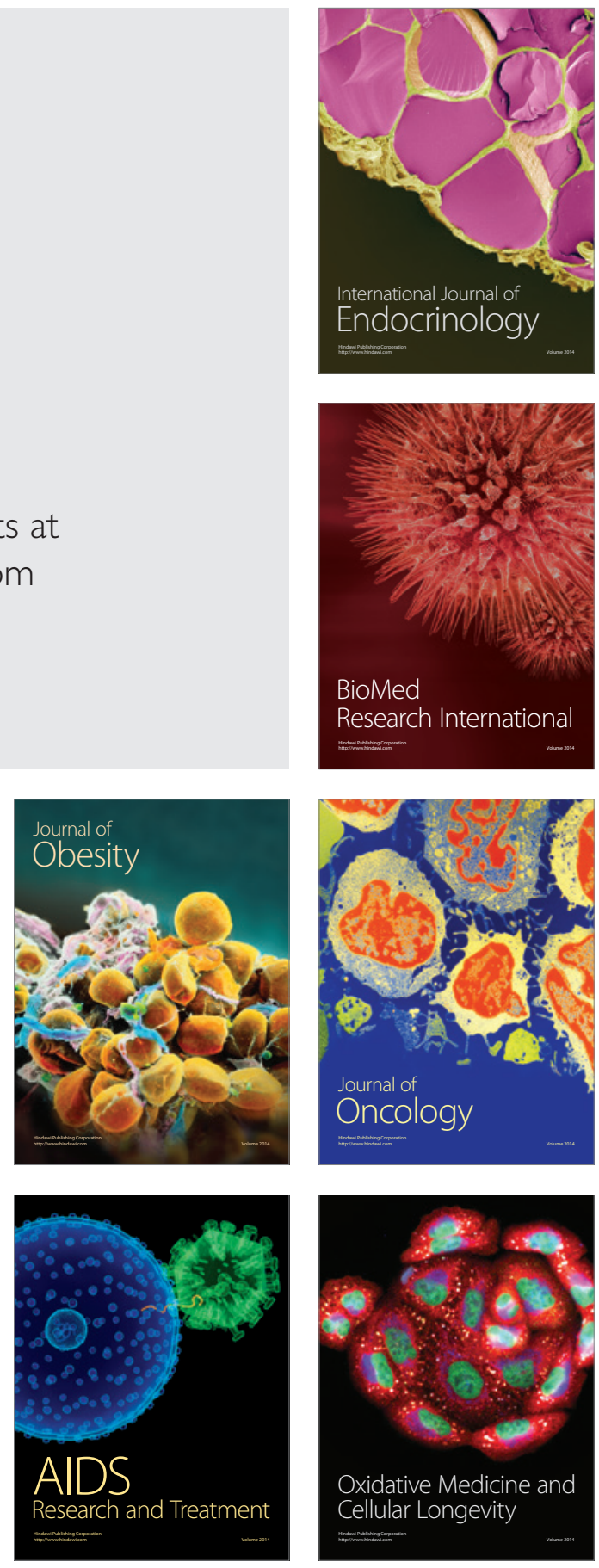